\title{
Kinder bewegen Stadt
}

Choreografische Praxis kann ganz allgemein als körperlich-sinnliche Wissensproduktion in Bewegung beschrieben werden. Ob dieses flüchtige, oft nur schwer oder gar nicht mit Worten beschreibbare Wissen für eine Praxis der kritischen Stadtforschung nutzbar gemacht werden kann, ist bisher nicht ausreichend erforscht. Dieser Text versucht eine praktische Annäherung aus künstlerischer Perspektive und ist gleichzeitig eine Einladung an die Disziplin der kritischen Stadtforschung, sich dem Themenfeld anzunähern.

Das Verständnis dessen, was choreografische Praxis eigentlich bedeutet oder bedeuten kann, wurde im letzten Jahrzehnt des tanzwissenschaftlichen Diskurses stark von einem „erweiterten Choreografiebegriff“ (Barthel 2015: 140) geprägt. Weit über die Beobachtung und Ausführung tänzerischer Bewegung hinausgehend wird Choreografie dabei als „Organisation von Körpern in Zeit und Raum“ (Klein 2012: 25) verstanden. Damit sind auch nicht-menschliche Körper und Materialien gemeint. Die Kulturwissenschaftlerin Heike Lüken bringt in diesem Kontext in Bezug auf städtische Räume den Begriff der „choreografischen Stadtforschung“ins Spiel. Diese definiert sie als eine „Praxistheorie“, die „ästhetische, also körperlich sinnliche Wissensproduktion in Bewegung, von Bewegungsund Wahrnehmungspraktiken und deren Materialisierung im Stadtraum“ (Lüken 2015: 154 ff.). Es geht also darum, einen Ort über den Körper und die Sinne zu begreifen, zu ertasten und zu erspüren. Dabei wird die eigene Wahrnehmung geschärft und das dadurch neu erlangte Wissen unmittelbar und über Bewegung an denselben Ort zurückgespielt. Es ist ein lernender, ergebnisoffener, nonverbaler ortsspezifischer Dialog, eine choreografische Praxis, die städtische Räume gleichzeitig schreibt und beschreibt.

Kinder begegnen städtischen Räumen spielerisch, voller Fragen und durch intensive Beobachtung. Besonders die Kleinsten eignen sich jede Umgebung systematisch durch Bewegung und Berührung an. Mit diesem besonderen Zugang zu ästhetischer Wissensproduktion verstehe ich in diesem Beitrag Kinder im Sinne der oben skizzierten choreografischen Stadtforschung als Expert_innen für Raumaneignung durch Bewegung. Im Folgenden beobachte ich sowohl typische Bewegungs- und Wahrnehmungsmuster von jüngeren 
Kindern im Alter zwischen zwei und sechs Jahren im Dialog mit städtischen Räumen als auch Kinder und Jugendliche, die sich explizit in tänzerischen oder choreografischen Kontexten bewegen.

In fünf persönlichen Gesprächen mit Personen aus den Arbeitsfeldern Pädagogik, Tanz/Choreografie, Kunstvermittlung und kulturelle Bildung konnte ich eine Reihe von Handlungen oder Herangehensweisen an räumliche Interaktionen beobachten, die ich im Sinne eines „sich in Beziehung Setzens“ als Schlüsselfähigkeiten von Kindern und Jugendlichen in Bezug auf die Aneignung städtischer Räume beobachtet habe.

Diese Beobachtungen - nicht nur von Kindern, sondern vor allem von Erwachsenen - können auch als direkte Handlungsaufforderungen zu forschenden, körperlich-sinnlichen Interaktionen mit städtischen Räumen gelesen werden. Aus den folgenden elf Abschnitten des Beitrags entsteht am Ende des Textes ein Bewegungsscore, eine Partitur von Handlungsanweisungen für das Erkunden von Stadt durch Bewegung. Der Text wird damit im Sinne des beschriebenen erweiterten Choreografiebegriffs selbst zur Choreografie. Gleichzeitig lädt er die erwachsenen Leser_innen dieses Beitrags zu einem ganz praktischen Perspektivenwechsel ein.

\section{Zeitlos und zweckfrei unterwegs sein}

Der im Wrangelkiez in Berlin-Kreuzberg ansässige Kinderladen „Cuvrybande e. V." legt einen Schwerpunkt seines pädagogischen Konzepts auf das freie Spiel. Die Erziehungspersonen halten sich so weit wie möglich zurück, um den Kindern ein möglichst freies Entscheiden über den Ort, Inhalt und zeitlichen Rahmen ihres Spiels zu ermöglichen (vgl. Pausewang 1997).

Die Kinder im Alter zwischen zwei und sechs Jahren verbringen täglich viel Zeit mit ihren Erzieher_innen in der unmittelbaren Nachbarschaft des Kiezes am Görlitzer Park. In einem Gespräch mit den Erzieher_innen des Kinderladens frage ich nach der Art, in der sich die Kinder mit ihrer Umgebung in Beziehung setzen, wenn sie dort unterwegs sind. Die Befragten führen aus, wie intensiv die Kinder auch in öffentlichen Räumen in ihr Spiel vertieft sind, wie zeitlos und zweckfrei sie unterwegs sind:

„Die vergessen ihr Rundherum; setzen sich auf die Straße und fangen an, Nüsse zu knacken. Fühlen sich völlig sicher und aufgehoben. Egal, ob auf dem Spielplatz oder auf der Straße. Sie sind dann in ihrer Welt. [...] Die haben ja auch alle Zeit, also die denken das zumindest. Und wenn sie mit uns unterwegs sind, haben sie das eigentlich auch-“ (Gespräch mit Katja Hüther (Gruppeninterview) am 18.12.2020)

\section{Sich ausstreuen}

Den Erzieher_innen fällt auch auf, dass die Kinder „sich ausstreuen“, sich also bei Spaziergängen räumlich weit verteilen, um sich Orte oder Strecken in ihrem eigenen Tempo anzueignen. Sie lieben es, „hinterherzutrödeln“ oder „vorzulaufen“(Gespräch mit Sunja Lee (Gruppeninterview) am 18.12.2020). Die Erzieher_innen lassen dies zu, vereinbaren aber mit den Kindern Treffpunkte, an denen sie sich immer wieder sammeln und aufeinander warten. 


\section{Einfach losrennen}

Der Tänzer und Choreograf Gabriel Galindez Cruz leitet seit fast zehn Jahren die Kindercompany von Sasha Waltz \& Guests mit bis zu 6o Kindern und Jugendlichen in vier verschiedenen Gruppen im Alter zwischen fünf und 17 Jahren.

Während des ersten Lockdowns der Coronapandemie im Frühling 2020 verlagert Galindez Cruz die Proben der Company nach draußen. Zwei Gruppen mit älteren Kindern finden einen festen Probenort im Tiergarten. Zwei Tänzerinnen der Company, Gemma Fiedler (11 Jahre alt) und Nika Brovot (12) sind begeistert vom neuen Probenort im Freien - so viel Platz, so viel frische Luft: „Man konnte sich ausbreiten, einfach losrennen“(Gespräch mit Nika Brovot am 20.1.2021). Umgeben von Bäumen, Gras und Büschen erweitern die Kinder durch Improvisationsaufgaben, Klettern und Krabbeln ihr Bewegungsrepertoire und machen aus der unmittelbaren Umgebung einen eigenen Forschungs- und Probenort unter freiem Himmel.

\section{Einem Ort zuhören}

Für eine Probe auf der Museumsinsel gibt Galindez Cruz neue Impulse für die Improvisationen: Die jungen Tänzer_innen sollen Orte finden, mit denen sie sich verbunden fühlen und dort in eine offene Bewegungsimprovisation gehen. Der Fokus liegt darauf, sich über den eigenen Körper mit diesem Ort „in einen Dialog zu begeben“, ihn also genau zu beobachten und auf das, was sie sehen, hören oder spüren, spontan mit Bewegung zu reagieren. Dabei entstehen detailreiche Improvisationen, aus denen kleine Solos oder Gruppenchoreografien werden, bei denen sich die Tänzer_innen gegenseitig zuschauen (Gespräch mit Gabriel Galindez Cruz am 9.12.2020).

\section{Sich unvermittelt begegnen}

Einige Passant_innen bleiben stehen, stellen Fragen, sind berührt oder verwundert. Diese Beobachtenden - ein anonymes öffentliches Publikum scheinen die Tänzer_innen nicht besonders zu stören: „Das war eigentlich kein Thema“ (Gespräch mit Nika Brovot am 20.1.2021). „Erst war mir das ziemlich peinlich, aber dann war es ganz normal und ich habe nicht weiter darüber nachgedacht“" (Gespräch mit Gemma Fiedler am 3.2.2021).

Galindez Cruz reflektiert den ungewöhnlichen und unmittelbaren Kontext dieser Begegnungen im Stadtraum:

„Die Menschen sprechen uns an: ,Was ist das, was macht Ihr da?‘ Eine Gruppe von Teenagern in der Stadt, [die] machen super konzentriert total verrückte Sachen mit ihren Körpern, [...] sie tanzen einfach, gehen ganz tief nach innen. Ich glaube, das ist wirklich nicht normal für dieses Alter." (Gespräch mit Gabriel Galindez Cruz am 9.12.2020)

Das Tanztraining von Galindez Cruz ermöglicht Kindern und Jugendlichen weit über das Kleinkindalter hinaus - einen offenen und spielerischen Zugang zu ihrer Umgebung. Sie sind virtuose Bewegungs- und Wahrnehmungsexpert_innen geworden und treffen in diesem Beispiel aufgrund der veränderten 
Trainingsbedingungen während der Pandemie ganz unvermittelt auf die Stadtgesellschaft. Nicht ganz unwichtig ist dabei, dass sie dieses Wissen auf für diese Altersgruppe ungewöhnlich selbstverständliche Weise mit den zufällig anwesenden Passant_innen teilen. Dabei beeinflussen sie wiederum deren ästhetische, also körperlich-sinnliche Wahrnehmung des Stadtraums.

\section{Entschleunigen}

Die Choreografin Sabine Zahn arbeitet im Januar 2015 in der belebten Umgebung des Berliner U-Bahnhofs Eberswalder Straße in Kooperation mit dem Projektträger „Theater Tusch“ mit einer Gruppe Grundschüler_innen zum Thema „Zeit und Bewegung“. Dabei wird intensiv beobachtet, gezeichnet und gesammelt sowie einzelnen Passant_innen gefolgt. Die spielerisch umgesetzte Rolle des „heimlich Beobachtenden“ erlaubt dies den Kindern beinahe nebenbei, indem sie den Alltagsbewegungen anderer Personen folgen und sich so selbst den Raum durch Bewegung aneignen (Gespräch mit Sabine Zahn am 23.12.2020).

Als finale Intervention entscheiden sich die Kinder für einen slow motion walk von ihrer Basis im Prater, einem ehemaligen Theater der Volksbühne, die Kastanienallee entlang bis zum U-Bahnhof Eberswalder Straße. Sämtliche Betreuungspersonen bleiben dabei im Hintergrund, damit die Gruppe von außen möglichst als unabhängig wahrgenommen werden kann. Beinahe eine Stunde braucht die 20-köpfige Gruppe, um in kompletter Stille und vollkommener Entschleunigung die Distanz von etwa 50 Metern zu überwinden. Zahn beschreibt im persönlichen Gespräch die verwirrten, erfreuten und entgeisterten Gesichter der Passant_innen, die dieser von Kindern erdachten und ausgeführten radikalen choreografischen Aneignung der Kastanienallee beiwohnen dürfen. Besonders bemerkenswert ist für sie das intuitive Entscheidungsvermögen und die kollektive Orientierung der jungen Menschen als Gruppe. In einem Gespräch im Anschluss an die Intervention thematisieren die Kinder, dass sie durch die Verlangsamung auch selbst für das Thema Geschwindigkeit sensibilisiert wurden (Gespräch mit Sabine Zahn am 23.12.2020).

\section{Details entdecken}

Die intensive Wahrnehmung von Details ist ein Beobachtungspunkt, der sowohl den Erzieher_innen der „Cuvrybande“ als auch Gabriel Galindez Cruz und Sabine Zahn immer wieder auffällt und die in allen Altersgruppen ausgeprägt zu sein scheint. Die Erzieherin Charlotte Rogmans beschreibt den ständig suchenden Blickjüngerer Kinder auf den Boden und in die Schaufenster, die dort Details entdecken, die keinem Erwachsenen auffallen würden (Gespräch mit Charlotte Rogmans (Gruppeninterview) am 18.12.2020). Sabine Zahn beschreibt, wie Grundschüler_innen sich auch auf einer belebten und lauten Straße ungewöhnlich lange und intensiv mit kleinsten Insekten beschäftigen (Gespräch mit Sabine Zahn am 23.12.2020). Gabriel Galindez Cruz bemerkt, dass den Jugendlichen beim Tanztraining Details wie die Bewegungen des Sonnenlichts oder die Beschaffenheit des Bodens auffallen (Gespräch mit Gabriel Galindez Cruz am 9.12.2020). 


\section{Sich Verankern}

Sabine Zahn ist davon überzeugt, dass Kinder sich an ihren Lieblingsorten „verankern“ (Gespräch mit Sabine Zahn am 23.12.2020), indem sie immer wieder die gleichen Handlungen vornehmen. Ihr natürliches Bedürfnis, durch Wiederholung zu lernen, macht Handlungsabläufe im Raum sichtbarer und beeinflusst so auch das sensible Geflecht räumlicher Interaktionen. Geht man davon aus, dass auch Kinder im Sinne der Raumproduktion nach Michel De Certeau durch ihre Handlungen städtische Räume hinterfragen, neu-definieren und damit verändern können (De Certeau 1988: 218), so ist dies eine räumliche Praxis, die sich durch ihr selbstbestimmtes, wiederholtes und aufmerksames „Praktizieren“ in der Umgebung „verortet“:

„Wenn wir als Erwachsene dem beiwohnen können, das ändert auch schon unsere Wahrnehmung, selbst wenn wir selbst nicht so entschleunigt sein können, oder so viele Details wahrnehmen können. [...] Der Fakt, dass Kinder an solchen Orten selbstverständlich sind und das praktizieren dürfen, das ändert den Raum. Die müssen das machen dürfen." (Gespräch mit Sabine Zahn am 23.12.2020)

Zahn beschreibt mit diesen Worten eine forschende und wiederholte Form von Bewegung, die ihre Umgebung bewegt: Eine eigene räumliche Praxis, durch die Kinder einen wichtigen Beitrag zum sozialräumlichen Gefüge der Stadtgesellschaft leisten.

\section{Schleichwege finden}

In Zusammenarbeit mit einem Team von Künstler_innen gründet Sabine Zahn im Oktober 2017 an der Nürtingen-Grundschule in Berlin-Kreuzberg die temporäre „Agentur für Analoge Raumspionage“ (Zahn 2017). Siefolgt der Aufmerksamkeit der Kinder für Orte, die diese sehr gut kennen: Was beobachten Kinder? Wie tun sie das? Wie können Orte durch Bewegung (neu) entdeckt oder entdeckt werden? Angefangen auf dem eigenen Schulhof und von dort aus weiter gestreut in die direkte Umgebung, werden Beobachtungen gesammelt, gezeichnet, archiviert und diskutiert. Die Kinder finden für die ihnen bekannten Orte immer wieder eigene Namen und verknüpfen diese mit den unterschiedlichsten Narrativen.

Die Kinder suchen sich unabhängig von den Aufgabenstellungen des Projekts immer wieder neue Schleichwege und Abkürzungen durch bekanntes Terrain (Gespräch mit Sabine Zahn am 23.12.2020). Bei anderer Betrachtung können diese spontanen Ab- und Umwege der Kinder aus der Perspektive der Choreografie als Raumkompositionen angesehen werden, als ästhetisch motivierte, kreative Bewegungen in Zeit und Raum, die einen selbstbestimmten Prozess der Aneignung dieser Orte beschreiben.

Die Vorstellungskraft der Kinder, die neue Nutzungen und Narrative auf bereits bekannte Orte projiziert, überlappt und verwebt sich mit dem bewusst erfahrenen, praktizierten Raum der Schleichwege und dem Wissen der Kinder über die repräsentative Funktion der sie umgebenden Architektur. Die Kinder entwickeln und bespielen damit ein bewegliches Puzzle aus verschiedenen Wahrnehmungsfeldern im Sinne des von Henri 
Lefebvre entworfenen Bildes der Dreiheit aus erfahrenem, vorgestelltem und beschriebenem Raum (Lefebvre 2006: 335-336).

\section{Schlammige Schätze suchen}

Die von „raumlaborberlin“ 2018 als temporäre urbane Forschungsstruktur gebaute „Floating University“, schwebt wie ein Luftschiff über dem Wassersammelbecken des Tempelhofes Feldes. Verwildert, versteckt und dennoch mitten in der Stadt, bietet dieser Ort endloses Potenzial für Geschichten und Abenteuer - für Kinder und Erwachsene gleichermaßen.

Zusammen mit der Szenografin und Kulturvermittlerin Ute Lindenbeck veranstaltet Sabine Zahn in der „Floating Kids Uni“ seit Sommer 2018 Workshops mit Kindern und Jugendlichen im Alter von sechs bis 16 Jahren. Die Teilnehmenden experimentieren - mit Lärm, schlammigen Schätzen, verschiedenen Materialien, Spaziergängen und kartografischen Übungen (Floating University 2020). Zahn und Lindenbeck beschäftigen sich dabei immer wieder mit der Frage, was Kinder tun, um sich einen Ort anzueignen sowie mit der Frage, welche ihrer räumlichen Praktiken in einer breit aufgestellten künstlerischen urbanen Forschungspraxis (für Erwachsene) fehlen (Gespräch mit Sabine Zahn am 23.12.2020). Diese Herangehensweise impliziert, die Interessen, Sichtweisen und Neugierde der Kinder und Jugendlichen zu Leitprinzipien einer forschenden künstlerischen Arbeit zu machen. Darüber hinaus schafft die „Floating University“ als temporärer, weitgehend ungestalteter Ort selbst die Möglichkeit einer besonders ergebnisoffenen selbstbestimmten Lernerfahrung.

\section{Einen Ort bespielen}

Das Netzwerk „Berlin Mondiale“ fokussiert sich darauf, Kunst und künstlerische Praxis für alle Stadtbewohner_innen gleichermaßen zugänglich und erlebbar zu machen (Gespräch mit der Sabine Kroner (Leitung „Berlin Mondiale“) am 7.1.2021). In Zusammenarbeit mit Künstler_innen, Vermittler_innen und verschiedenen Akteur_innen aus Stadtentwicklung und Kulturpolitik arbeitet es prozesshaft, interdisziplinär und soweit wie möglich mit allen Beteiligten auf Augenhöhe. Ein Schwerpunkt liegt dabei auf Möglichkeiten der Begegnung für marginalisierte Kinder und Jugendliche sowie auf Projekten im breiten Kontext von Migration, Exil und Asyl.

Im Pandemiesommer 2020 verlagert sich ein Großteil der Aktivitäten der „Mondiale“ zwangsläufig nach draußen. Dabei werden viele Freiflächen mit kulturellen Bildungsangeboten und Begegnungsformaten bespielt, wie etwa das sogenannte Wasserwerk auf dem Gelände einer Kirche in der Nähe des Moritzplatzes in Berlin-Kreuzberg. Dieses wird in den Sommermonaten zu einem Begegnungsort der umliegenden Wohnsiedlungen. Hier sind es vor allem offene, unkomplizierte und non-verbale Spielformate für Kinder und Jugendliche, wie sie von Gabriel Galindez Cruz durchgeführt wurden, die besonders gut angenommen werden (Gespräch mit Gabriel Galindez Cruz am 9.12.2020). Die Theaterpädagogin und Theaterwissenschaftlerin Laura Werres koordiniert und betreut die kulturellen Projekte der „Mondiale“-Standorte von 
Spandau bis Marzahn inhaltlich und organisatorisch. Den ganzen Sommer ist sie draußen unterwegs an den verschiedenen Knotenpunkten des Netzwerks und beobachtet mit Begeisterung, wie Projekte mit jungen Menschen sich öffentliche Räume „erobern“ und dabei ganz aktiv in Interaktion mit der Stadtgesellschaft gehen: „Das hat so viel Potenzial, man besetzt einfach so den Raum. Alles spielt mit, alles wird Teil des Projekts." (Gespräch mit Laura Werres am 7.1.2021) Einig sind sich Laura Werres und Sabine Kroner, die seit 2015 die „Berlin Mondiale“ leitet, jedoch darin, dass es die Selbstverständlichkeit, mit der ältere Kinder und Jugendliche vielleicht noch vor ein paar Jahrzehnten städtische Räume und Freiräume für sich selbst in Anspruch genommen haben, heute nicht mehr gibt. Ihrer Erfahrung nach braucht es hierfür eine explizite Einladung und längere vertrauensbildende Prozesse durch soziokulturelle Akteur_innen und Kulturvermittler_innen (Gespräch mit Sabine Kroner und Laura Werres am 7.1.2021).

\section{Fazit}

Die ästhetische Wahrnehmungs- und Forschungsexpertise von Kindern und Jugendlichen bringt detailreiche, ebenso zeitlose wie ergebnisoffene Beobachtungen, ausgeprägte Improvisationsfähigkeit sowie fragende, spielerische Begegnung und Intervention in den Alltag städtischer Räume. Sie ist damit ein wertvoller Beitrag zum Beobachten, Verstehen und sinnlichen Begreifen von Stadt.

Choreograf_innen und Tanzvermittler_innen wie Gabriel Galindez Cruz und Sabine Zahn arbeiten systematisch daran, mit Wahrnehmungs- und Bewegungsforschung „,kindliche“ Zugänge zur Raumaneignung zu erhalten oder sich diese neu bewusst zu machen. Sie trainieren den Körper und die Sinnevon Kindern, Jugendlichen und auch Erwachsenen als Forschungsinstrumente, mit denen das sensible und hochdynamische Konstrukt des Raums, der uns umgibt, gelesen und dessen Wahrnehmung auf unterschiedlichsten Ebenen verstärkt werden kann. Prinzipiell sind Vermittler_innen wie Galindez Cruz und Zahn zwar in der Lage, jedem Menschen Zugang zu diesem bewegten Wissen zu ermöglichen, die Realität sieht jedoch oft anders aus. Tänzerische und choreografische Praxen sind bisher weder einer breiten Stadtgesellschaft zugänglich noch für Forschungsfelder wie die kritische Stadtforschung wirklich nachvollziehbar oder übersetzbar. Ergebnisse aus der Praxis haben demnach nur exemplarischen Charakter - wie auch die in diesem Beitrag geschilderten Ergebnisse. Sie werden nur selten analysiert und verbalisiert und bleiben so subjektive Erfahrungen und flüchtige Interaktionen. In ihrer Flüchtigkeit bespielen sie einen städtischen Ort zwar temporär und reflektieren ihn auch kritisch, erzielen aber keine langfristige Wirkung.

In einem ersten Schritt der Annäherung zwischen den Disziplinen der Choreografie und der kritischen Stadtforschung lade ich die Leser_innen deshalb hier und jetzt ein, sich zunächst selbst durch das körperlich-sinnliche „praktizieren“ der folgenden, direkt aus dem Text entstandenen, choreografischen Übungen auf einen ersten unmittelbaren Wissenstransfer einzulassen, der die Ergebnisse dieses künstlerischen Forschungsbeitrags in die eigene Praxis übersetzt. 


\section{Einladung zu einer choreografischen Stadtforschungsübung}

Künstlerisches Format: Bewegungsscore/Partitur

1. Zeitlos und zweckfrei unterwegs sein

2. Sich ausstreuen

3. Einfach losrennen

4. Einem Ort zuhören

5. Sich unvermittelt begegnen

6. Entschleunigen

7. Details entdecken

8. Sich verankern

9. Schleichwege finden

10. Schlammige Schätze suchen

11. Einen Ort bespielen

Hinweis: Jede einzelne dieser Handlungsaufforderungen kann für sich alleine im Stadtraum praktiziert und durch Wiederholung verankert werden. Kombinationen sind in der vorgegebenen oder in jeder beliebigen Reihenfolge möglich. Die Dauer dieser choreografischen Übungsfolge ist frei wählbar und kann bei jeder Wiederholung der Partitur verändert werden.

\section{Autor_innen}

Gabriele Reuter ist Choreografin und Urbanistin. Sie beschäftigt sich in künstlerischen, pädagogischen, partizipativen und planerischen Kontexten mit den Themen Stadtraum und Bewegung.

post@gabrielereuter.de

\section{Literatur}

Barthel, Gitta (2015): Choreografische Praktiken im öffentlichen Raum. Vom Interaktionsgefüge der Partizipanden. In: Marianne Bäcker / Mechthild Schütte (Hg.), Tanz - Raum - Urbanität. Jahrbuch Tanzforschung. Leipzig: Henschel, 137-146.

de Certeau, Michel (1988 [1980]): Die Kunst des Handelns. Berlin: Merve. (Übers. Ronald Voullié)

Floating University (2020): Infotext zur Floating Kids Uni. https://www.floatinguniversity. org/kinder-uni/ (letzter Zugriff am 15.2.2021).

Lefebvre, Henri (2006 [1974]): Die Produktion des Raums. In: Jörg Dünne / Stephan Günzel (Hg.), Raumtheorie. Frankfurt am Main: Suhrkamp, 335-336. (Übers. Jörg Dünne)

Klein, Gabriele (2012): Choreografien des Alltags. Die emergenten Ordnungen der Lebenswelt. In: Claudia Behrens / Helga Burkhard / Claudia Fleischle-Braun / Krystyna Obermaier (Hg.), Tanzerfahrung und Welterkenntnis. Jahrbuch Tanzforschung. Leipzig: Henschel, 17-33.

Lüken, Heike (2015): Choreografische Stadtforschung. Wissensproduktion in Bewegung. In: Marianne Bäcker / Mechthild Schütte (Hg.), Tanz - Raum - Urbanität. Jahrbuch Tanzforschung. Leipzig: Henschel, 147-158.

Pausewang, Freya (1997): Dem Spielen Raum geben. Grundlagen und Orientierungshilfen zur Spiel- und Freizeitgestaltung in sozialpädagogischen Einrichtungen. Berlin: Cornelsen.

Zahn, Sabine (2017): Kiezspione 17. http://www.lovelabours.net (letzter Zugriff am 15.2.2021). 


\section{Interviews/persönliche Gespräche:}

Gruppeninterview mit den Erzieher_innen des Kinderladens „Cuvrybande e. V.“ in BerlinKreuzberg (Charlotte Rogmans, Katja Hüther, Sunja Lee, Chris Dunkel) am 18.2.2020.

Sabine Zahn, persönliches Gespräch am 23.12.2020.

Gabriel Galindez Cruz, persönliches Gespräch am 9.12.2020.

Nika Brovot, persönliches Gespräch am 20.1.2021.

Gemma Fiedler, persönliches Gespräch am 3.2.2021.

Sabine Kroner und Laura Werres („Berlin Mondiale“), persönliches Gespräch am 7.1.2021. 
